\title{
OPTIMUM AERODYNAMIC SHAPE DESIGN FOR FLUID FLOW PROBLEMS INCLUDING MESH ADAPTIVITY
}

\author{
G. BUGEDA* AND E. OÑATE \\ Escola Tècnica Superior d'Enginyers de Camins, Canals i Ports, Universitat Politècnica de Catalunya, \\ C/ Gran Capità s/n, Campus Nord UPC, 08034 Barcelona, Spain
}

\begin{abstract}
SUMMARY
This paper presents a methodology for solving shape optimization problems in the context of fluid flow problems including adaptive remeshing. The method is based on the computation of the sensitivities of the geometrical design parameters, the mesh, the flow variables and the error estimator to project the refinement parameters from one design to the next. The efficiency of the proposed method is checked out in two 2D optimization problems using a full potential model coupled with a boundary layer model. The first one corresponds to an internal flow problem and the second one corresponds to an external flow problem. The work presented here can be considered as a continuation of previous work (Bugeda and Oñate, Int. J. Numer. Methods Fluids, 20, 915-924 (1995)). In that paper, the sensitivity analysis corresponding to both an incompressible potential flow and a Euler flow were derived together with a strategy for the adaptive remeshing. In the present paper, the sensitivity analysis is derived for a full potential flow coupled with a boundary layer model, and a new error estimator is employed. Copyright (C) 1999 John Wiley \& Sons, Ltd.
\end{abstract}

KEY WORDS: shape optimization; adaptivity; potential flow; boundary layer

\section{INTRODUCTION}

The increasing complexity of fluid flow problems to be analyzed with the finite element method makes necessary the use of meshes with an adequate sizing of the elements adapted to the flow features. This is necessary to capture the flow complexities (shock waves and boundary layers) but it increases considerably the computational cost of the analysis.

The high cost of the numerical analysis of a complex flow can be reduced if an adaptive remeshing strategy is used. In this case small elements are used only in the zones where the flow is complex, whereas bigger elements are used in the rest of the domain. The basic requirements of this type of strategy are the following:

- a non-structured mesh generator able to control the sizes of the elements everywhere;

- an a posteriori error estimator or indicator to assess where smaller elements are needed;

- an optimality criteria for the definition of the characteristics (i.e. element sizing) of an optimal mesh for a given CPU cost, or a given precision.

* Correspondence to: Universitat Politècnica de Catalunya, C/ Gran Capità s/n, Campus Nord UPC, 08034 Barcelona, Spain.

Contract/grant sponsor: The EUROPT I project

Contract/grant sponsor: The ECARP project

CCC 0271-2091/99/100161-18\$17.50

Copyright (C) 1999 John Wiley \& Sons, Ltd. 
Adaptive remeshing procedures are typically based on a series of successive analysis of the flow problem using meshes with increasing quality. This is possible because the characteristics of the flow problem are the same during the successive analysis. This procedure is much more complex if these are continuously changing, as in the case of an optimization problem. Here a succession of different designs are obtained and the flow pattern can change from one design to the next. An 'optimal' mesh for the analysis of one design can become inadequate for the next one. This can be crucial if the location of flow discontinuities (i.e. shocks) changes from one design to the next.

Traditionally, optimization problems are solved iteratively, and a single mesh is properly adapted for the analysis of each geometry. There is no control on the quality of the results that can lead to convergence problems and also to bad final results. It should be noted that not only the quality of the analysis but also that of the sensitivities with respect to the design variables depend on the quality of the meshes.

To insert an adaptive remeshing loop within the optimum design iterative process is an obvious possibility. The problem is that in this case, the CPU cost of the problem grows proportionally to the number of optimization iterations multiplied by the number of adapted meshes used for each design.

In a standard adaptive remeshing process, the definition of a good mesh requires a previous analysis using a given mesh. In the iterative resolution of an optimum design problem different geometries are obtained and analyzed, but the fluid flow characteristics are different for each one. The authors [1] have recently proposed a methodology where the information required for each adaptive remeshing is taken from the analysis of the previous design. Both the mesh parameters and the error estimator are 'projected' to the next enhanced design. This allows $a$ priori enhanced (refined) meshes to be defined, which ensures improved quality of the results
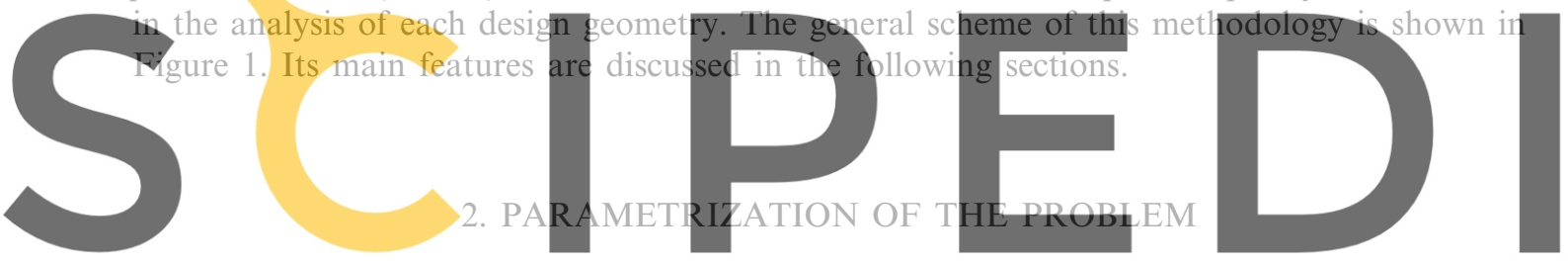

Each design geometry is represented by using 'definition points' that specify some interpolation

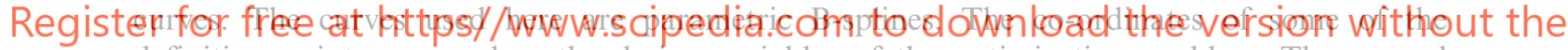
definition points are used as the design variables of the optimization problem. The general expression of a closed B-spline for $q$ points is [10]:

$$
\boldsymbol{r}(t)=\sum_{l=0}^{q} \boldsymbol{r}_{l} N_{4, l+1}(t)
$$

where $\boldsymbol{r}(t)$ is the position vector depending on a parametric variable $t$. The co-ordinates of the definition points are recovered using $t=0,1,2, \ldots$ The curve is expressed as a linear combination of $q+1$ normalized fourth-order (cubic) B-splines. The degree of continuity of a cubic B-spline is $C^{2}$. The $\boldsymbol{r}_{l}$ coefficients are the co-ordinates of the so-called polygon definition points and they are found by using the co-ordinates of the definition points and some additional conditions about slopes and curvatures to build up a linear system of equations.

The derivation of expression (1) with respect to the co-ordinates of the definition points provides the sensitivities of the co-ordinates of the boundary points with respect to the design variables. Details of this process can be found in [1]. 


\section{MESH GENERATION AND SENSITIVITY ANALYSIS}

In order to control the sizes of the elements, a non-structured mesh generation algorithm should be used. In this work, the well-known advancing front method has been chosen [3]. The characteristics of the mesh are specified via a background mesh over which nodal values of the size parameter $h$ are defined and interpolated via the shape functions. The background mesh for the first design has to be specified by the user. For subsequent designs, the background mesh is taken to coincide with the mesh projected into this design from the previous one. This projection process will be described later.

Once the sensitivities of the co-ordinates of each boundary node are known, it is also possible to compute the sensitivities of the co-ordinates of each internal nodal point (mesh sensitivities). These sensitivities are necessary to assess how the mesh evolves when the design variables change.

There are many different ways to define the evolution of the mesh in terms of the design variables. It is possible to consider a simple analogous elastic medium defining the mesh movement. This is the basis of the so-called 'spring analogy', where each element side is regarded as a spring connecting two nodes. The force induced by each spring is proportional to its length and the boundary nodes are considered fixed. The solution of the equilibrium problem in the spring analogy is simple but expensive, and it requires solving a linear system of equations with two degrees of freedom per node.

In this work, the spring analogy problem has been solved iteratively using a simple Laplacian smoothing approach. This technique is frequently used to improve the quality of non-structured meshes. It consists of the iterative modification of the nodal co-ordinates of
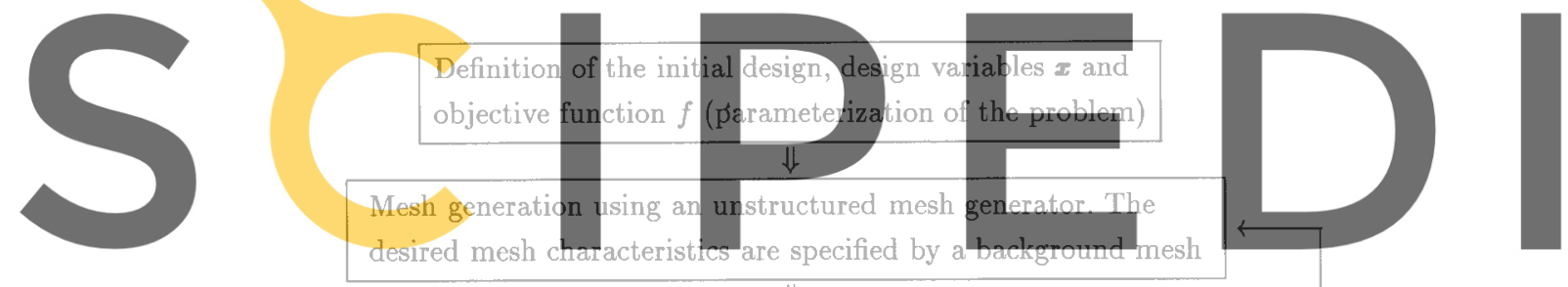

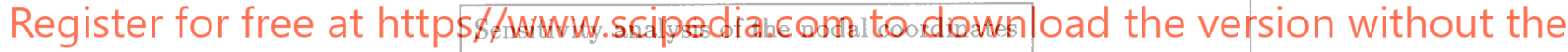

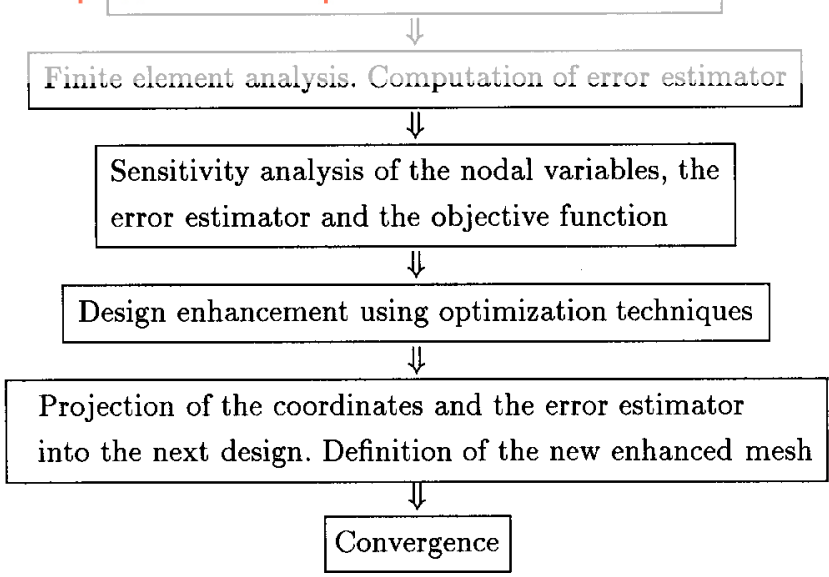

Figure 1. Proposed new optimum design methodology. 


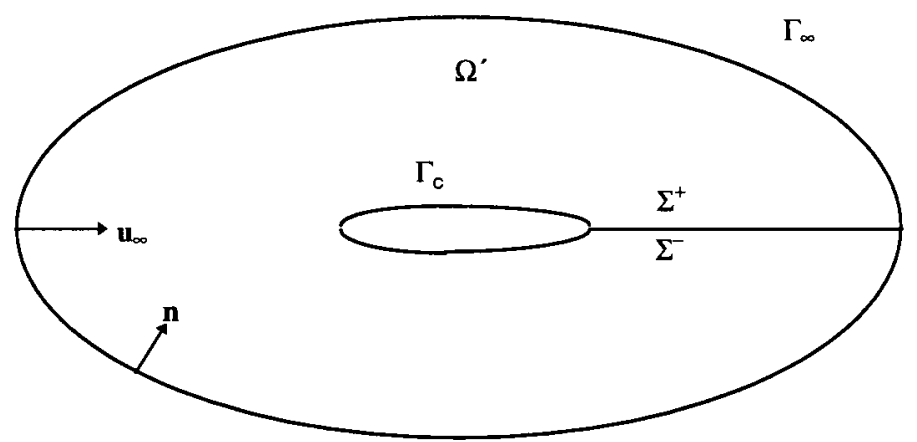

Figure 2. Definition of the fluid flow problem.

$$
\left\{\begin{array}{l}
c_{*}^{2}=\frac{\gamma-1}{\gamma+1}+\frac{2}{(\gamma+1) M_{\infty}^{2}}, \\
\rho_{*}=\left(M_{\infty}^{2} c_{*}^{2}\right)^{1 /(\gamma+1)}
\end{array}\right.
$$

where $M_{\infty}$ is the far-field Mach number, $\gamma$ is equal to 1.4 and $c$ is the actual sound velocity.

A Dirichlet boundary condition must also be added in order to define the zero potential level. The inlet part $\Gamma_{\mathrm{I} \infty}$ of the far-field boundary is chosen for this condition.

The weak formulation of Equation (4) must take into account the lift. Thus, a potential jump is taken on the wake $\Sigma$ by considering two dissociate lines $\Sigma^{+}$and $\Sigma^{-}$, which replace $\Sigma$.

$2+$ joins the upper surface of the airfoil with the far-field boundary and $\Sigma-$ the lower part of
the boundary with it. Then, the weak formulation is formed in the domain $\Omega$, whose boundary
is $\Gamma_{\infty} \cup \Gamma_{c} \cup \Sigma^{+} \cup \Sigma^{-}$. By multiplying (4) by a test function $w \in \boldsymbol{H}^{1}$ and integrating by parts,
on obtains the weak formulation:
find $\phi \in V_{0}\left(\Omega^{\prime}\right)$ such that $\forall w \in V_{0}\left(\Omega^{\prime}\right)$ :
\[ \int_{\Omega^{\prime}} \frac{\partial \rho}{\partial t} w \mathrm{~d} \Omega-\int_{\Omega^{\prime}} \rho \boldsymbol{u} \nabla w \mathrm{~d} \Omega+\int_{\partial \Omega_{0}^{\prime}} \rho \boldsymbol{u n w} \mathrm{d} \gamma-\int_{\partial \Omega^{\prime}} s \nabla\left(\delta s \frac{\partial F}{\partial s}\right) w \mathrm{~d} \Omega=0 \text {. } \]

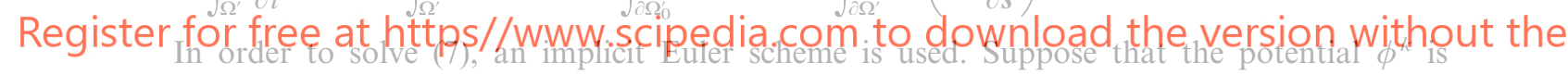
known at the $k$ th step. Then, the solution at the $(k+1)$ th step is given by the equation:

$$
\begin{aligned}
& \int_{\boldsymbol{\Omega}^{\prime}} \frac{\rho^{k+1}-\rho^{k}}{t^{k+1}-t^{k}} w \mathrm{~d} \Omega-\int_{\Omega^{\prime}} \rho^{k+1} \boldsymbol{u}^{k+1} \nabla w \mathrm{~d} \Omega+\int_{\partial \Omega_{0}^{\prime}} \rho^{k+1} \boldsymbol{u}^{k+1}+\boldsymbol{n} w \mathrm{~d} \gamma \\
& -\int_{\partial \boldsymbol{\Omega}^{\prime}} \boldsymbol{s} \nabla\left(\delta s \frac{\partial F}{\partial \boldsymbol{s}}\right) w \mathrm{~d} \boldsymbol{\Omega}=0,
\end{aligned}
$$

which can be rewritten as

$$
G\left(\phi^{k+1}\right)=\int_{\Omega^{\prime}} \frac{\rho^{k+1}-\rho^{k}}{t^{k+1}-t^{k}} w \mathrm{~d} \Omega+J\left(\phi^{k+1}\right)=0, \quad \forall w \in V_{0}\left(\Omega^{\prime}\right) .
$$

The velocity at the $k$ th time step is obtained by $\boldsymbol{u}^{k+1}=\boldsymbol{u}^{k}+\nabla\left(\phi^{k+1}-\phi^{k}\right)$ and the fluid density is a function of $\left|\boldsymbol{u}^{k+1}\right|$. Therefore, Equation (9) gives $\phi^{k+1}$ since $\phi^{k}$ is known. The space discretization is done by $\mathrm{P}^{1}$ finite elements. The steady state solution of Equation (9) is obtained by using the techniques described in [4]. 


\subsection{Sensitivity analysis of the full potential solver}

The sensitivity analysis of all the magnitudes involved in Equation (8) can be obtained by direct derivation of the integral expressions. The first step is the computation of the sensitivities of the potential $\phi$ with respect to any design variable $x$ (see $[2,11]$ ).

The term $J$ of Equation (9) depends on the potential $\phi$, and the design variable $x$. In the steady state it must hold that:

$$
J_{i}(x, \phi)=0, \quad i=1, \ldots, n,
$$

where $n$ is the number of trial functions used for the discretization of $\phi$. This expression can be derivated with respect to the design variable $x$ giving

$$
\frac{\partial J_{i}}{\partial x}+\frac{\partial J_{i}}{\partial \phi_{j}} \frac{\partial \phi_{j}}{\partial x}, \quad i, j=1, \ldots, n .
$$

Equation(s) (11) allow the sensitivities of the potential value at each node $\phi_{j}$ to be obtained with respect to $x$ as

$$
\frac{\partial \phi_{j}}{\partial x}=-\left[\frac{\partial J_{i}}{\partial \phi_{j}}\right]^{-1} \frac{\partial J_{i}}{\partial x} .
$$

\section{Note that this expression involves the resolution of a linear system of equations.}

Once the computation of the sensitivities of $\phi$ are formed, the sensitivities of any other magnitude (velocities, density, pressure, objective function, etc.) can be obtained by direct derivation of its expressions in terms of the potential and the design variables.

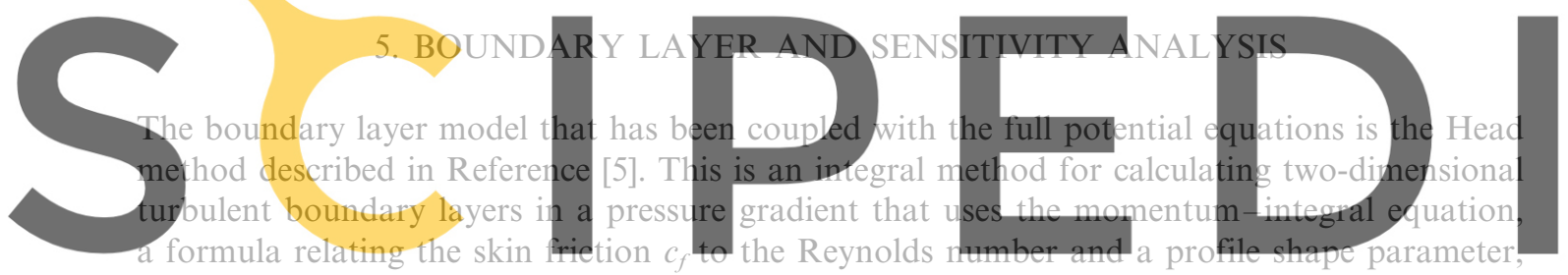

and an ODE for the rate of change of profile shape parameter with $x$.

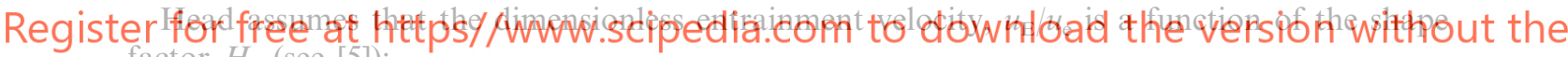 factor $H_{1}$ (see [5]):}

$$
\frac{u_{\mathrm{E}}}{u_{\mathrm{e}}}=\frac{1}{u_{\mathrm{e}}} \frac{\mathrm{d}}{\mathrm{d} x}\left[u_{\mathrm{e}}\left(\delta-\delta^{*}\right)\right]=F\left(H_{1}\right)
$$

where $u_{\mathrm{e}}$ is the external inviscid velocity, $\delta$ is the boundary layer thickness, $\delta *$ is the displacement thickness and $H_{1}$ is defined in terms of the momentum thickness $\Theta$ by

$$
H_{1}=\frac{\delta-\delta^{*}}{\Theta} \text {. }
$$

After substitution of Equation (14) into (13), one obtains

$$
\frac{\mathrm{d}}{\mathrm{d} x}\left(u_{\mathrm{e}} \Theta H_{1}\right)=u_{\mathrm{e}} F
$$

Head assumes that $H_{1}$ is related to the shape factor $H$ by

$$
H_{1}=G(H) \text {. }
$$


Functions $F$ and $G$ are determined from experiments. A best fit to several tests of experimental data (see [5]) is:

$$
\begin{aligned}
& F=0.0306\left(H_{1}-3.0\right)^{-0.6169}, \\
& G= \begin{cases}0.8234(H-1.1)^{-1.287}+3.3 & H \leq 1.6 \\
1.5501(H-0.6778)^{-3.064}+3.3 & H \leq 1.6 .\end{cases}
\end{aligned}
$$

The momentum integral equation can be written as

$$
\frac{\mathrm{d} \Theta}{\mathrm{d} x}+(H+2) \frac{\Theta}{u_{\mathrm{e}}} \frac{\mathrm{d} u_{\mathrm{e}}}{\mathrm{d} x}=\frac{c_{f}}{2} .
$$

This expression involves three unknowns: $\Theta, H$ and $c_{f}$ for a given external velocity distribution. Equation (15), with $F, H$ and $G$ defined by Equations (16)-(18) provides a relationship between $\Theta$ and $H$. Another equation relating $c_{f}$ to $\Theta$ and/or $H$ is needed. Head used the $c_{f}$ law given by Ludwieg and Tillmann:

$$
c_{f}=0.246 \times 10^{-0.678 H} \operatorname{Re}_{\Theta}^{-0.268},
$$

where $R e_{\Theta}=u_{\mathrm{e}} \Theta / v$ is the Reynolds number based on the momentum thickness and the kinematic viscosity $v$. The system of Equations (15)-(19), which includes two ODEs, can be solved numerically for a specific external velocity distribution to obtain the boundary layer development.

This method, like most integral methods, uses the shape factor $H$ as the criterion for Separation. Equation (19) predicts $\Phi_{f}=\Theta$ only if $H$ tends to infinity. It is no possible
to give an exact value of $H$ corresponding to separation, and a range between 1.8 and
2.4 is commonly quoted. The difference betyeen the lower and upper limits of $H$ makes
ony little difference in locating the separation point, since close to separation, $\mathrm{d} H / \mathrm{d} x$ is
large.
After the resolution of the system of ODEs, the Squire and Y oung formula is used for

an estimation of the total drag:

\section{Register for free atshttps//www.scipedia.com to download the version without the $c_{\mathrm{d}}=2 \frac{\bar{L}}{u_{\mathrm{E}}}$,

where $L$ is the total length of the boundary layer.

Reference [5] presents a Fortran program for predicting the turbulent boundary layer development on two-dimensional bodies by the method of Head. The system of equations is integrated by a Runge-Kutta scheme for a given set of initial conditions. This program has been implemented and tested, giving good results.

The sensitivity analysis of the Head method has been obtained by direct differentiation of all the expressions appearing in the Fortran code. This task has been developed with the MATHEMATICA program, which has helped for the differentiation of the most complex expressions. The MATHEMATICA program can provide, in Fortran, the derivative of an expression with respect to any of its symbols in terms of all the symbols that appear in the expression and its derivatives. The new code containing all the differentiated expressions provides the drag and its sensitivities with respect to the design variables. 


\section{ERROR ESTIMATOR AND SENSITIVITY ANALYSIS}

An 'energy' norm of the potential $\phi$ will be used as the reference magnitude that will control the adaptivity process. This norm is defined in terms of the exact velocities field $\boldsymbol{u}$ as:

$$
U=\|\phi\|^{2}=\int_{\Omega} \boldsymbol{u}^{T} \boldsymbol{u} \mathrm{d} \Omega
$$

The discretization process provides an approximated potential $\hat{\phi}$ with an associated velocity field $\hat{\boldsymbol{u}}$ whose 'energy' norm is

$$
\|\hat{\phi}\|^{2}=\int_{\Omega} \hat{\boldsymbol{u}}^{T} \hat{\boldsymbol{u}} \mathrm{d} \Omega .
$$

Last norm contains an error that for elliptic problems can be computed using the expression:

$$
\|e\|^{2}=\int_{\Omega}(\boldsymbol{u}-\hat{\boldsymbol{u}})^{T}(\boldsymbol{u}-\hat{\boldsymbol{u}}) \mathrm{d} \Omega .
$$

For non-elliptic problems, the last expression is assumed to be an approximation to the error in the 'energy' norm. The quality of this approximation depends of the relative magnitude of the non-elliptic terms in front of the elliptic ones.

Of course, expression (23) cannot be computed if the exact solution $\phi$ is not known. Then, the exact velocities $\boldsymbol{u}$ are replaced by an approximated velocities field $\overline{\boldsymbol{u}}$ that is supposed to be of better quality than the solution $\hat{\boldsymbol{u}}$. This replacement provides the value of the error estimator $\eta$ that can be computed element by element as:

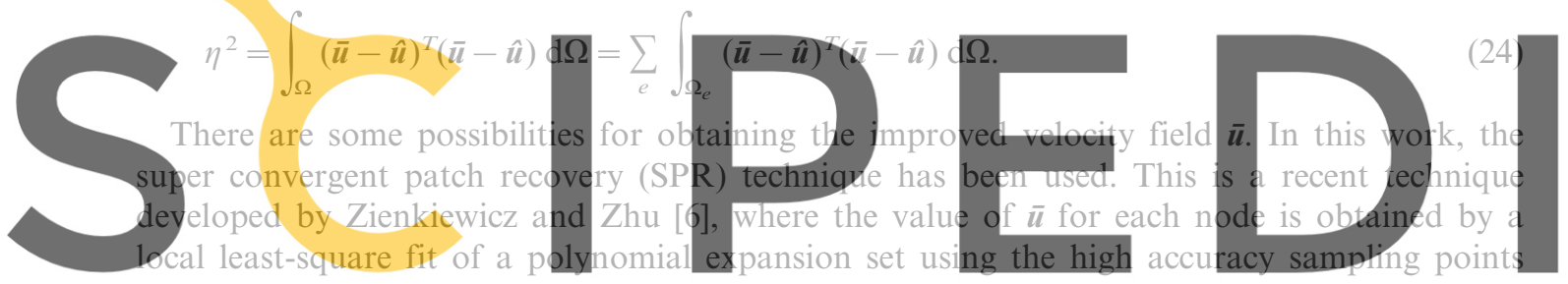

inside the element patch connected with the node. This procedure does not involve the

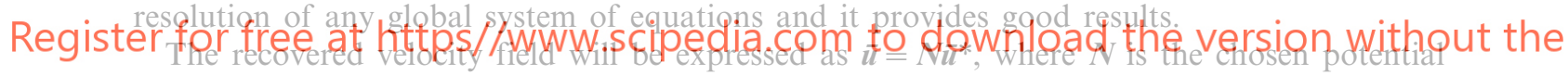

interpolation function and $\boldsymbol{u}^{*}$ is the recovered nodal value of the velocities. In the recovery process, the nodal value $\boldsymbol{u}^{*}$ is assumed to belong to a polynomial expansion $\boldsymbol{u}^{*}$ of the same complete order $p$ as that of the basis functions $\boldsymbol{N}$ and which is valid over an element patch surrounding the particular node considered. This polynomial expansion will be used for each component of $\boldsymbol{u}_{p}^{*}$ and it is simply written:

$$
\boldsymbol{u}_{p}^{*}=\boldsymbol{P a},
$$

where $\boldsymbol{P}$ contains the appropriate polynomial terms and $\boldsymbol{a}$ is a set of unknown parameters. For $\mathrm{P}^{1}$ triangular finite elements used in this work, one has $\boldsymbol{P}=[1, x, y]$.

The determination of the unknown parameters $\boldsymbol{a}$ is best made by ensuring a least-square fit to the set of high accuracy sampling points within the patch. The sampling points are the barycenters of the triangles forming the patch. To do this, the following must be minimized:

$$
F(\boldsymbol{a})=\sum_{i=1}^{n}\left(\hat{\boldsymbol{u}}\left(x_{i}, y_{i}\right)-\boldsymbol{u}_{p}^{*}\left(x_{i}, y_{i}\right)\right)^{2}=\sum_{i=1}^{n}\left(\hat{\boldsymbol{u}}\left(x_{i}, y_{i}\right)-\boldsymbol{P}\left(x_{i}, y_{i}\right) \boldsymbol{a}\right)^{2},
$$


where $\left(x_{i}, y_{i}\right)$ are the co-ordinates of the $n$ sampling points. The minimization condition of $f(\boldsymbol{a})$ implies that $\boldsymbol{a}$ satisfies:

$$
\sum_{i=1}^{n} \boldsymbol{P}^{T}\left(x_{i}, y_{i}\right) \boldsymbol{P}\left(x_{i}, y_{i}\right) \boldsymbol{a}=\sum_{i=1}^{n} \boldsymbol{P}^{T}\left(x_{i}, y_{i}\right) \hat{\boldsymbol{u}}\left(x_{i}, y_{i}\right) .
$$

This can be solved in matrix form as:

$$
\boldsymbol{a}=\boldsymbol{A}^{-1} \boldsymbol{b},
$$

where

$$
\begin{aligned}
\boldsymbol{A} & =\sum_{i=1}^{n} \boldsymbol{P}^{T}\left(x_{i}, y_{i}\right) \boldsymbol{P}\left(x_{i}, y_{i}\right), \\
\boldsymbol{b} & =\sum_{i=1}^{n} \boldsymbol{P}^{T}\left(x_{i}, y_{i}\right) \hat{\boldsymbol{u}}\left(x_{i}, y_{i}\right) .
\end{aligned}
$$

In order to get the sensitivities of the error estimator it is necessary to obtain the sensitivities of the recovered nodal velocities. These will be obtained by direct derivation of Equations (25), (28) and (29):

$$
\begin{aligned}
& \frac{\partial \boldsymbol{u}_{p}^{*}}{\partial \boldsymbol{d}}=\frac{\partial \boldsymbol{P}}{\partial \boldsymbol{d}} \boldsymbol{a}+\boldsymbol{P} \frac{\partial \boldsymbol{a}}{\partial \boldsymbol{d}}, \\
& \frac{\partial \boldsymbol{P}}{\partial \boldsymbol{d}}=\left[0, \frac{\partial x}{\partial \boldsymbol{d}^{\prime}}, \frac{\partial y}{\partial \boldsymbol{d}}\right] .
\end{aligned}
$$

(31)

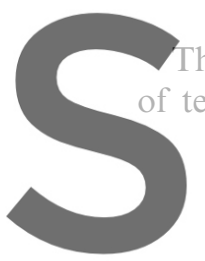

The terms $\partial x / \partial d$ and $\partial y / \partial d$ can be of terms it holds that:

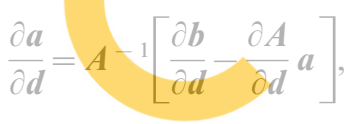

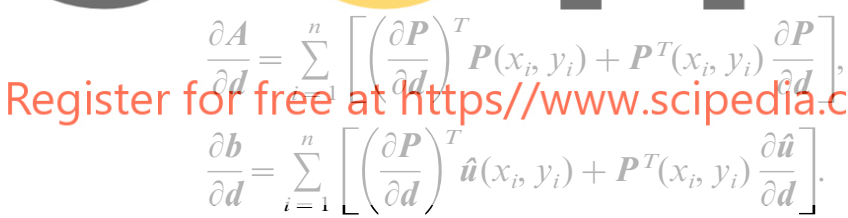
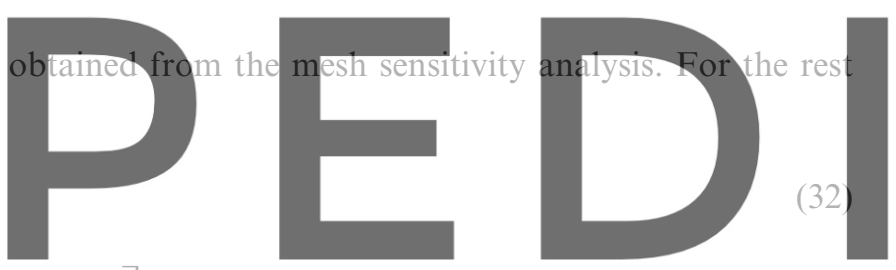

The sensitivities of the velocities at the barycenters of the elements $\hat{\boldsymbol{u}}$ will be obtained from the flow sensitivity analysis. The sensitivities of the recovered velocities $\overline{\boldsymbol{u}}$ will be obtained from

$$
\frac{\partial \overline{\boldsymbol{u}}}{\partial \boldsymbol{d}}=\boldsymbol{N} \frac{\partial \overline{\boldsymbol{u}}^{*}}{\partial \boldsymbol{d}}=\boldsymbol{N} \frac{\partial \boldsymbol{u}_{p}^{*}}{\partial \boldsymbol{d}}
$$

The sensitivities of the error estimator $\eta^{2}$ can be obtained by direct derivation of the integral expression (24). The sensitivities of an integral expression are computed after its transformation into the isoparametric domain $\xi$, whose shape does not depend on the design variables. The Jacobian of this transformation $|\boldsymbol{J}|$ can be expressed in terms of the nodal co-ordinates, so that it can also be differentiated in order to obtain the integral sensitivities. Using this technique, the sensitivities of the integral expression of the error corresponding to each element $e$ can be obtained as: 
Once the new design has been obtained, all the relevant variables for the adaptive remeshing strategy can be projected from the old design to the new one using the sensitivity analysis. Second-order sensitivities can be used if required. The co-ordinates of the projected mesh are obtained by

$$
(x, y)_{k+1} \approx(x, y)_{k}+\theta\left(\frac{\partial x}{\partial \boldsymbol{s}}, \frac{\partial y}{\partial \boldsymbol{s}}\right)+\frac{1}{2} \theta^{2}\left(\frac{\partial^{2} x}{\partial \mathbf{s}^{2}}, \frac{\partial^{2} y}{\partial \mathbf{s}^{2}}\right) .
$$

For the case of incompressible potential equations, the projections of the error estimator and the 'energy' norms can be obtained from:

$$
\begin{aligned}
& \|e\|_{k+1}^{2}=\|e\|_{k}^{2}+\theta \frac{\partial\|e\|^{2}}{\partial \boldsymbol{s}}+\frac{1}{2} \theta^{2} \frac{\partial^{2}\|e\|^{2}}{\partial \boldsymbol{s}^{2}}, \\
& \|U\|_{k+1}^{2}=\|U\|_{k}^{2}+\theta \frac{\partial\|U\|^{2}}{\partial \boldsymbol{s}}+\frac{1}{2} \theta^{2} \frac{\partial^{2}\|U\|^{2}}{\partial \boldsymbol{s}^{2}} .
\end{aligned}
$$

These projections provide a good approximation of each of above values for the next design configuration previously to any new computations. In fact, the projected values provide the necessary information to perform a remeshing over the next design, even before any new computation is attempted. In that sense, an error estimator computed a posteriori has been changed into an a priori error estimator for the definition of a new mesh.

This projection is of paramount importance since it allows the quality of the mesh for each new design to be controlled. Only a single mesh is generated in every new design analysis step. Thus, the extra computational cost involved in the control of the mesh quality is very small.

The projected values are used to create the background mesh information needed to generate the mesh corresponding to the new design geometry. This operation closes the iterative process that leads to the optimum design geometry after convergence.

\section{NUMERICAL RESULTS}

This section contains results for the solution of two different problems. The first one corresponds to the minimization of the viscous drag of a nozzle. The second one is the solution of an airfoil reproduction problem including viscous effects.

\subsection{Nozzle viscous drag minimization}

In this problem, the optimization of a simple symmetric 2D nozzle geometry under subsonic flow conditions is considered. The inlet and outlet cross-sections and the inlet-to-throat area ratio of the nozzle are fixed acting as geometrical constraints in the optimization process. The cost function is defined as a global measure of the viscous drag along the solid walls in the area of interest $0 \leq x \leq 2$. The nozzle is symmetric with respect to the $y=0$ line (see Figure 3 ). The area of interest lies between $y=0$ and $x=2$. The half-height of the inlet and outlet sections is $y_{\max }=0.5$ and the half-height of the throat is $y_{\mathrm{thr}}=0.35$. The Mach number is 0.2 and the Reynolds number based on half-length $(l=1)$ is $2 \times 10^{6}$. The computational domain is defined as $-2 \leq x \leq 4$ for inflow and outflow uniformity reasons. The upper boundary of the geometry outside the area of interest is fixed $\left(y=y_{\max }\right)$.

The purpose of the problem is to find the nozzle shape satisfying the geometrical constraints and minimizing the viscous drag on the solid walls in the area of interest. The boundary layer development is considered in the area of interest only, starting with turbulent initial conditions 


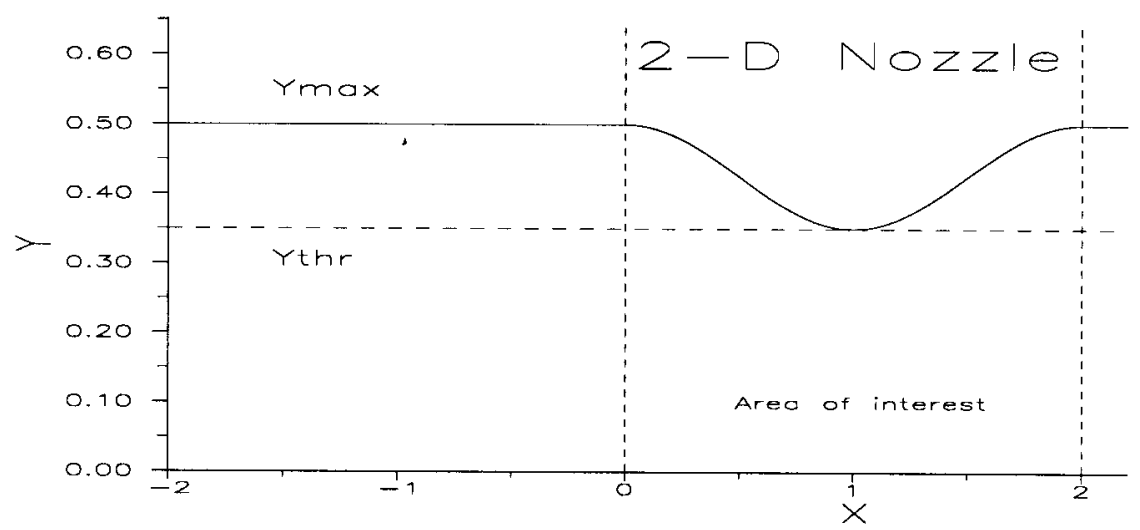

Figure 3. Definition of the nozzle problem.

at $x=0$, in order to avoid incompatibilities due to the laminar-to-turbulent transition criterion. The initial boundary layer conditions are:

$$
\delta / l=4.97 \times 10^{-3}, \quad \delta^{*} / l=8.64 \times 10^{-4}, \quad H=\delta_{1} \Theta=1.431 .
$$

The initial shape corresponding to the first design in the optimization process is given by (see Figure 3):

$$
0 \leq x \leq 2 ; \quad y=a_{0}+a_{1} \sin (\pi(x-1.5)), \quad a_{0}=\left(y_{\max }+y_{\text {thr }}\right) / 2, \quad a_{1}=\left(y_{\max }+y_{\text {thr }}\right) / 2 .
$$

This problem has been solved by using the methodology previously described. Figure 4 shows the initial shape of the nozzle and the mesh used for the analysis of this initial design. Linear triangles have been used for the discretization of the domain. The limit of the allowable global percentage of error $\gamma$ (see expression (38)) has been fixed to $0.02 \%$.

The objective function is the total drag of the nozzle $\left(C_{\mathrm{D}}\right)$. This has been obtained by using the Square and Young formula (see expression (20)), where the boundary layer properties are computed at $x=2$ and $u$ is the local inviscid velocity. The velocities obtained from the full potential model have been used for the computation of the boundary layer model by using the Head method.

Four design variables have been used for the definition of the shape of each design. This variables are the $y$ co-ordinates of four points placed on the upper surface. The $x$ co-ordinates of these points are $0.333,0.667,1.333$ and 1.667 respectively. The $y$ co-ordinate corresponding to $x=1.000$ is 0.35 , as specified in the definition of the test case.

After 100 iterations, no significant improvements have been obtained in the shape of the nozzle. Figure 5 shows the shape of the final design and the mesh used for its analysis. As can be observed, a big concentration of elements has been produced in the vicinity of the nozzle.

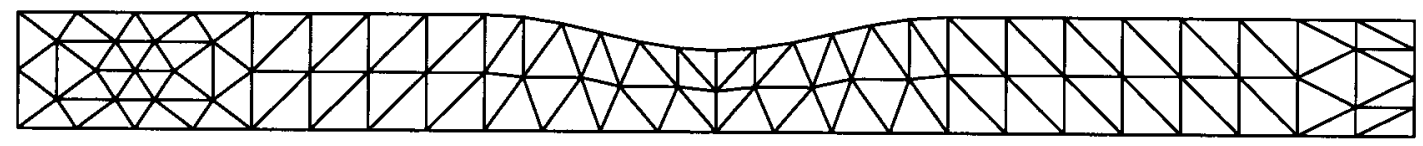

Figure 4. Initial shape and initial mesh with 124 elements and 92 nodes. 


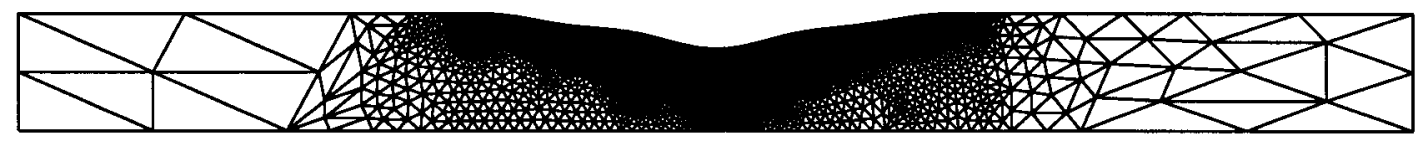

Figure 5. Final shape and final mesh with 7136 elements and 3719 nodes.
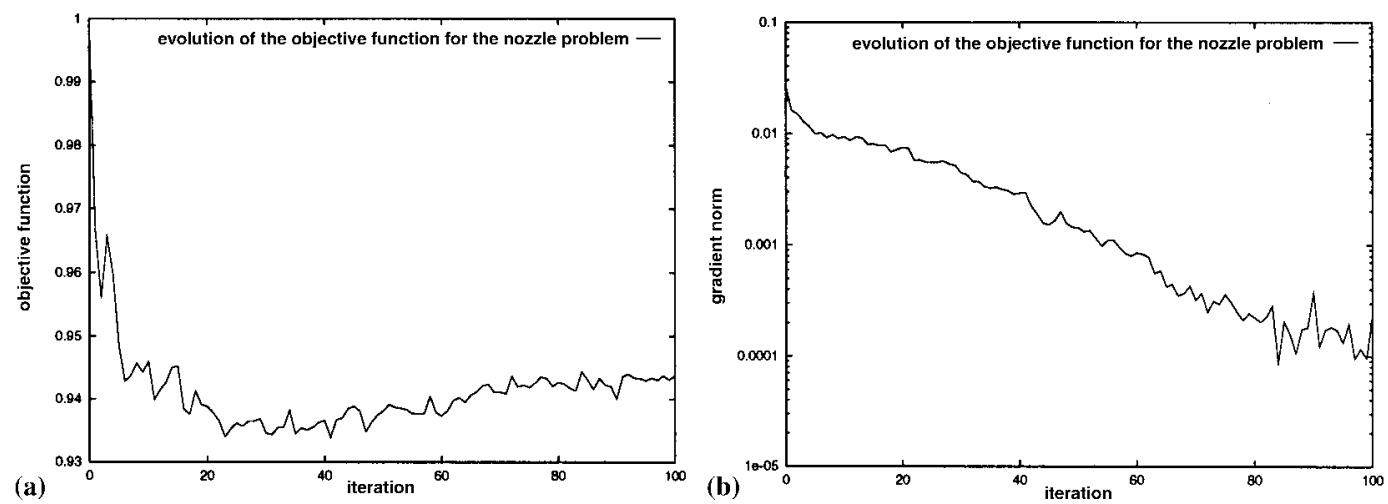

Figure 6. (a) Evolution of the objective function; (b) evolution of the norm of the gradient of the objective function.

Figure 6 shows the evolution of the objective function and the norm of its gradient during the iterative process. It can be observed how, after the initial 40 iterations, the objective function does not diminish. Nevertheless, the gradient norm is minimized until the 80th iteration. This is probably due to the mesh refinement process, which during the last iterations and without apparent changes in the design and the objective function, provides better values for the norm of the gradient of the objective function. The process continues the iterative optimization scheme if an improvement of the norm of the gradient is detected.

The oscillations that are present in the evolution of both the objective function and its gradient norm are probably due to the change of the mesh for each different design. These oscillations are small if a very small global percentage of error $\gamma$ is prescribed. Bigger values of $\gamma$ produce cheaper meshes, but the oscillations in the iterative process are bigger and the convergence is worst. Figure 7 shows how the global percentage of error is maintained below the $0.02 \%$ value prescribed for the generation of the mesh for each design.

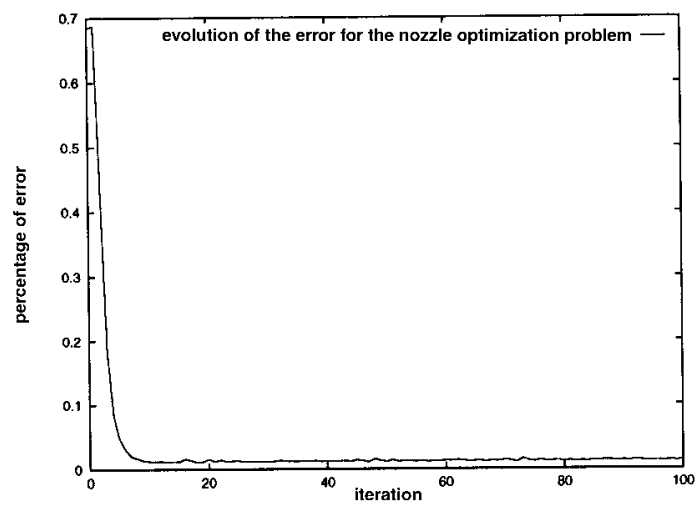

Figure 7. Evolution of the global percentage of error. 

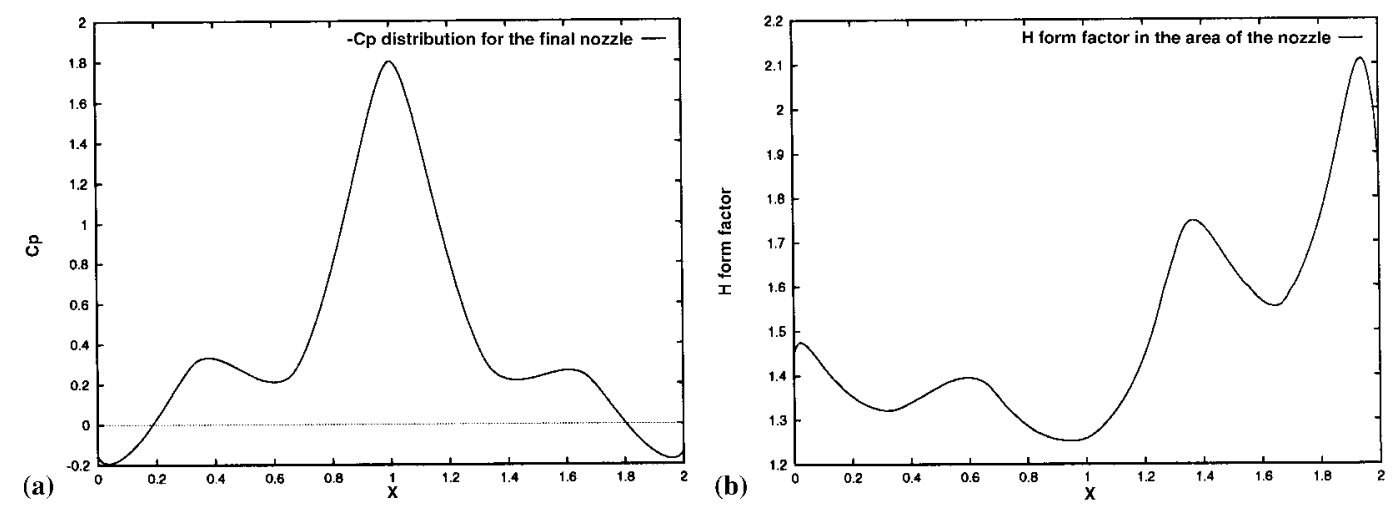

Figure 8. (a) $C_{p}$ distribution in the area of the nozzle; (b) $H$ form factor in the area of the nozzle.

Figure 8 shows the $C_{p}$ distribution in the area of the nozzle. This distribution is not smooth as it can be expected from Figure 5. Also, Figure 8 shows a non-smooth distribution of the $H$ form factor of expression (13). In fact, the values of the $H$ form factor show that in some parts of the nozzle, the boundary layer is separating. This fact makes the boundary layer model chosen inappropriate for the analysis of the final design. However, from the mathematical point of view, the model is good enough to check the goodness of the presented methodology. A more elaborated resolution of this problem should include a restriction on the separation of the boundary layer.

9.1.1. Analysis of the results. The study of the results for this problem allows the following comments to be made:

- The results obtained by using the presented methodology including mesh adaptivity show the good behavior of the method. The obtained final optimum shape is unexpected, but it is in accordance with the final solutions presented by other authors in a workshop held at INRIA Sophia Antipolis in the context of the ECARP Brite-Euram project [9]. The reason of this unexpected optimum shape seems to be the use of the Square and Young expression, which can provide unrealistic results for this type of geometry. On the other hand, as it has been mentioned, there is no control on the possible separation of the boundary layer.

- The quality of the meshes used for the analysis of each design is well-controlled, and therefore, the good quality of the results obtained for each design is assured.

- In order to get a good convergence of the iterative process, it is necessary to use a very strict limit in the global percentage of error. In fact, it has been observed that the use of poor quality meshes considerably reduces the cost of the analysis of each design but, on the other hand, it produces big difficulties in converging to a final solution. The reason is that poor meshes produce not only bad quality results but also bad quality sensitivities.

\subsection{Airfoil reproduction problem}

This problem concerns the reproduction of an airfoil under viscous conditions. The objective of the problem is the reproduction of a NACA 0012 profile from the 'target pressure' distribution, which corresponds to this profile at $M_{\infty}=0.3$, incidence $\alpha=3^{\circ}$ and $\operatorname{Re}=3 \times 10^{6}$ (based on chord $c$ ). The starting point for the design process is the NACA 63215 airfoil. 
The methodology used for the resolution of this test case is exactly the same as described for the previous example. This test case is an inverse problem where the difference between a target $C_{p_{\text {target }}}$ distribution and the $C_{p}$ distribution corresponding to a given design is used as an objective function. Due to the thin boundary layer obtained at $R e=3 \times 10^{6}$ and assuming that the pressure distribution is constant across the boundary layer, a week coupling has been used between the inviscid full potential model and the boundary layer equations. The procedure for this coupling is as follows:

- The velocities are computed by using the full potential model.

- The boundary layer equations are solved by using the inviscid velocities around the profile. This provides the boundary layer thickness at each point of the profile.

- For each point of the profile, the $C_{p}$ coefficient is evaluated at a distance equal to the boundary layer thickness from the profile.

The target distribution, $C_{p_{\text {target }}}$, has been obtained by a direct analysis of the NACA 0012 profile using linear triangular elements and an adaptive remeshing scheme with a maximum global percentage of error $\gamma$ fixed to $0.01 \%$. The objective $F$ function has been computed as:

$$
F=\int\left(C_{p}-C_{p \text { target }}\right)^{2} \mathrm{~d} s
$$

This function has been normalized by dividing last expression by the value obtained for the initial design. In this way, the objective function obtained for each design is a normalized measure of the difference between its corresponding pressure distribution and the target one. The optimum design will be that providing a zero value of the objective function.

Figure 9 shows the initial shape of the profile corresponding to a NACA 63215 and the mesh used for the analysis of this initial design. Linear triangles have been used again for the discretization of the domain. The limit of the allowable global percentage of error $\gamma$ has been fixed to $0.1 \%$.

Fifteen design variables have been used for the definition of the shape of each design. This variables are the $y$ co-ordinates of seven points on the upper surface, seven points on the lower surface and the leading edge. The seven points on both the upper and lower surfaces are distributed in a sinusoidal form, with a bigger concentration of points in the leading and trailing edges.

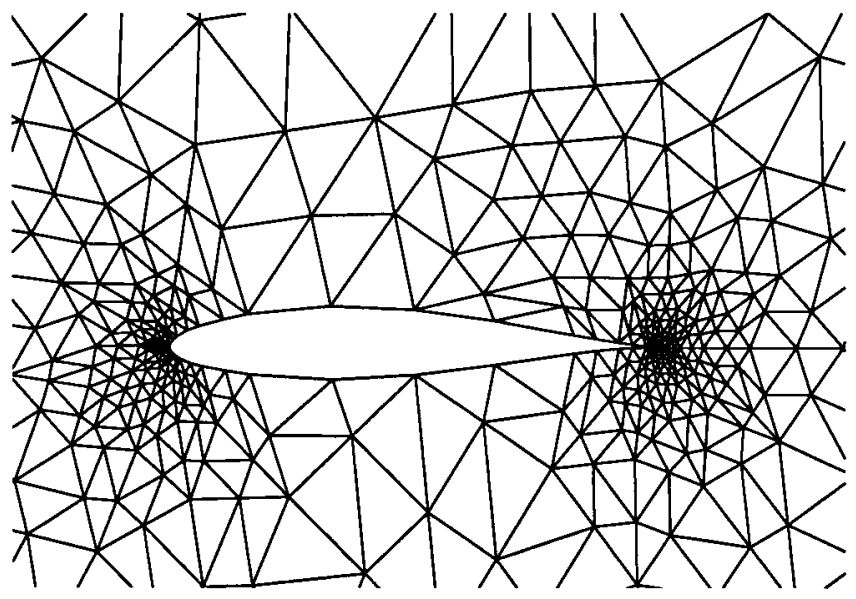

Figure 9. Initial shape and initial mesh with 920 elements and 1736 nodes. 


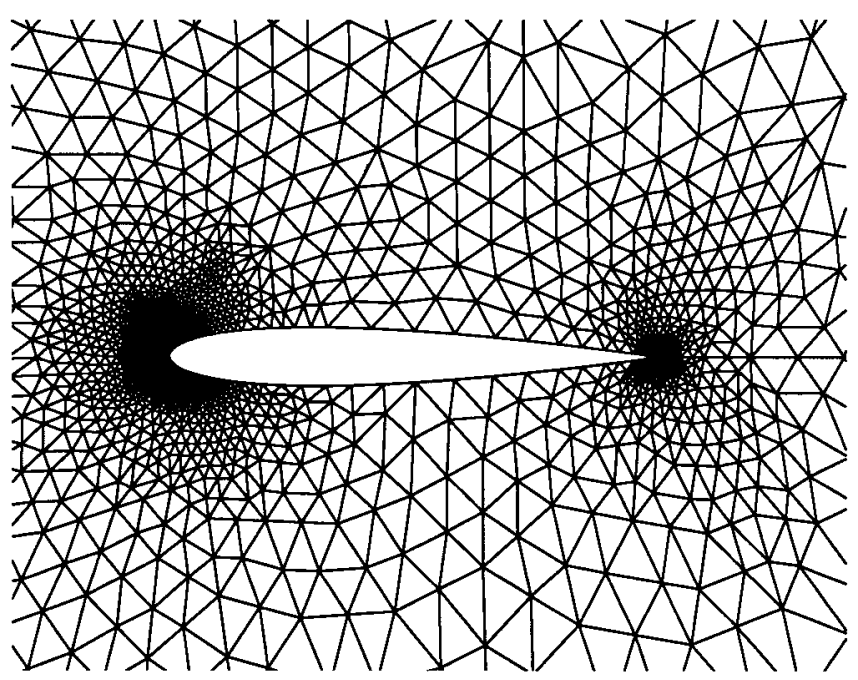

Figure 10. Final shape and final mesh with 6124 elements and 3149 nodes.

Figure 10 shows the shape and the mesh of the final design obtained after 100 iterations. Figure 11 shows the evolution of the objective function during the iterative process. After 100 iterations, there is no significant modification of the objective function and it has been considered to be converged. Nevertheless, the objective function has converged to a value slightly higher than 0.0 as it is normally expected for a reproduction problem. There are two reasons for this difference:

- The target pressure has been computed by a direct analysis of the NACA 0012 airfoil and using a complete definition of its geometry with more than 100 points. As the reconstruction process uses 'only' 15 design variables, it is not possible to recover the same exact geometry as the one defined with a higher number of points.

- The direct analysis of the NACA 0012 airfoil has been performed with an adapted unstructured mesh. Even in the case that it was possible to recover the exact NACA 0012 geometry, the mesh used for the analysis would be different than the one used for the direct
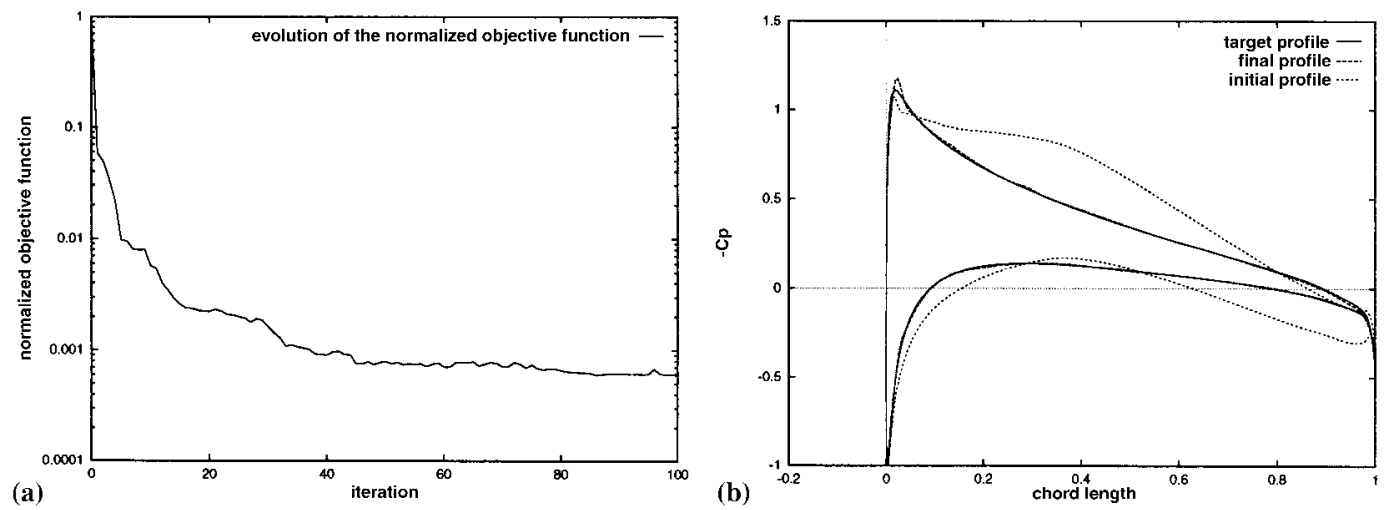

Figure 11. (a) Evolution of the normalized objective function; (b) superposition of the initial, final and target airfoil profiles. 


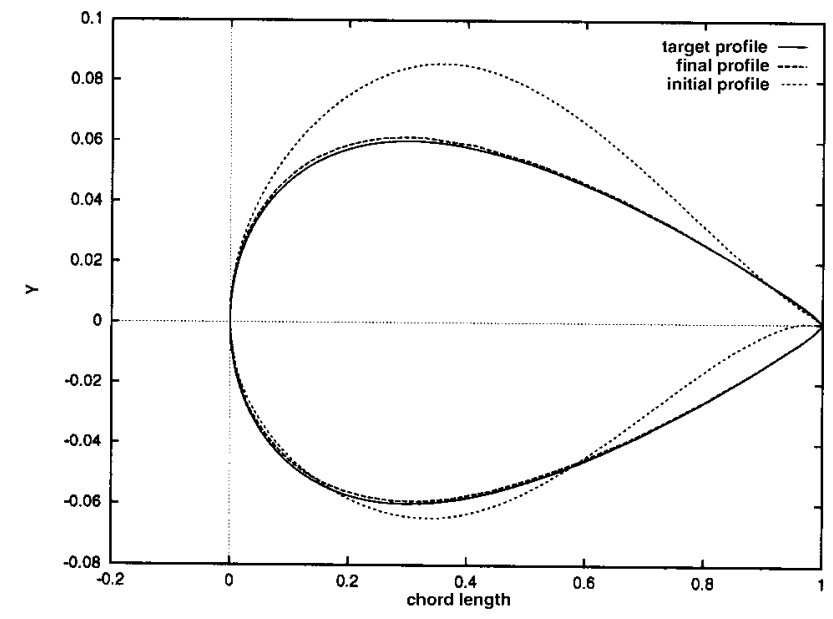

Figure 12. Superposition of the initial, final and target $C_{p}$ geometries.

analysis and it would produce a small difference between the reconstructed pressure and the target one.

Figure 11 also shows the superposition of the $C_{p}$ distributions corresponding to the initial, final and target profiles. Excellent agreement between the final and the target profiles is obtained taking into account that only 15 points have been used for the geometrical definition of the reconstructed profile.

Figure 12 shows the superposition of the geometries corresponding to the initial, final and target profiles. Here also the agreement between the final and the target profiles is almost perfect.

9.2.1. Analysis of the results. The conclusions from this test case are similar to those obtained with the previous one:

- A very good agreement between the target and the final designs is obtained. Nevertheless, the quality of the convergence process is very influenced by the parameterization. In fact, the number of points used for the definition of the geometries is critical. This is because the use of a small number of design variables does not allow a final design close to the target one to be achieved. On the other hand, the use of too many design variables considerably increases the computational cost.

- The quality of the meshes used for the analysis of each design is well-controlled and the good quality of the results obtained for each design is assured.

- In order to get a good convergence of the iterative process, it is necessary to use a very strict limit in the global percentage of error. Here, the same comments as in the previous problem about the quality of the meshes can be made.

\section{CONCLUSIONS}

A new methodology for the resolution of aerodynamic shape optimization and inverse problems has been developed and assessed. This methodology allows the design and the analysis mesh to be optimized in a joint manner in order to produce a final design computed with a proper mesh. 
Good quality results are obtained using a single mesh for each design without any remeshing. This considerably reduces the additional cost of the mesh control.

The presented methodology has provided excellent results for all the cases analyzed leading to an accurate final solution with a good final mesh.

The use of a single and different 'optimal' meshes for each optimum design step seems to be specially interesting for the solution of realistic flow problems where the control of the mesh quality is crucial. This could be particularly attractive in presence of shocks or separation points. Further assessment of this methodology in the context of both Euler and NavierStokes flows is in progress [1] and will be reported in the near future.

\section{ACKNOWLEDGMENTS}

This work was partially funded by the EUROPT I and ECARP projects in the Brite-Euram Aeronautics program of the EEC.

\section{REFERENCES}

1. G. Bugeda and E. Oñate, 'Optimum aerodynamic shape design including mesh adaptivity', Int. J. Numer. Methods Fluids, 20, 915-924 (1995).

2. F. Beux, A. Dervieux, M.P. Leclercq, and B. Stoufflet, 'Techniques de contrôle optimal pour l'optimisation de forma en aérodynamique avec calcul exact du gradient', Revue Sci. Tech. Défense, 1994.

3. J. Peraire, K. Morgan and J. Peiró, 'Unstructured finite element mesh generation and adaptive procedures for CFD', AGARD FDP: Specialist's Meeting, Loen, Norway, 1989.

4. O. Pironneau, Finite Element Methods for Fluid, Wiley, New York, 1989.

5. T. Cebeci and P. Bradshaw, Momentum Transfer in Boundary Layer, Hemisphere, New York, 1977.

6. O.C. Zienkiewicz and J.Z. Zhu, 'The super convergent patch recovery and a posteriori error estimates. Part 1: the recovery technique', Int. J. Numer. Methods Eng., 33, 1331-1364 (1992).

7. G. Bugeda and E. Oñate, 'New adaptive techniques for structural problems', 1st Europ. Conf. on Numerical Methods in Engineering, Brussels, September, 1992.

8. E. Oñate and G. Bugeda, 'A study of mesh optimality criteria in adaptive finite element analysis', Eng. Comput., 10, 307-321 (1993).

9. J. Periaux et al. (eds.), Notes on Numerical Fluid Mechanics. Volume 61. Optimum Aerodynamics Design \& Parallel Navier-Stokes Computations. ECARP_European Computational Aerodynamics Research Project, Vieweg, Berlin, 1998.

10. I.D. Faux and M.J. Pratt, Computational Geometry for Design and Manufacture, Ellis Horwood Limited, 1987.

11. A. Vossinis, 'Optimisation de forme d'aile d'avion', Doctoral Thesis, University of Paris VI, 1993. 